\title{
Malignant pleural effusion: from bench to bedside
}

\author{
Ioannis Psallidas ${ }^{1}$, Ioannis Kalomenidis², Jose M. Porcel ${ }^{3}$, \\ Bruce W. Robinson ${ }^{4,5}$ and Georgios T. Stathopoulos 6,7 \\ Number 1 in the Series "Pleural Diseases" \\ Edited by Najib Rahman and loannis Psallidas
}

\begin{abstract}
Affiliations: ${ }^{1}$ Oxford Respiratory Trials Unit, Oxford Centre for Respiratory Medicine, Oxford University Hospitals Trust, Oxford, UK. ${ }^{2} 1$ st Dept of Critical Care and Pulmonary Medicine, National and Kapodistrian University of Athens, School of Medicine, Evangelismos Hospital, Athens, Greece. ${ }^{3}$ Pleural Medicine Unit, Dept of Internal Medicine, Arnau de Vilanova University Hospital, Biomedical Research Institute of Lleida, Lleida, Spain. ${ }^{4}$ National Centre for Asbestos Related Disease, School of Medicine and Pharmacology, University of Western Australia, Perth, Australia. ${ }^{5}$ Dept of Respiratory Medicine, Sir Charles Gairdner Hospital, Nedlands, Australia. 'Laboratory for Molecular Respiratory Carcinogenesis, Dept of Physiology, Faculty of Medicine, University of Patras, Achaia, Greece. ${ }^{7}$ Comprehensive Pneumology Center (CPC), University Hospital, LudwigMaximilians University and Helmholtz Zentrum München, Member of the German Center for Lung Research (DZL), Munich, Germany.
\end{abstract}

Correspondence: Ioannis Psallidas, Oxford Centre for Respiratory Medicine, Oxford University Hospitals Trust, Old Road, Churchill site, OX3 7LN, Oxford, UK. E-mail: ioannis.psallidasandm.ox.ac.uk

ABSTRACT Malignant pleural effusion (MPE) is a common but serious condition that is related with poor quality of life, morbidity and mortality. Its incidence and associated healthcare costs are rising and its management remains palliative, with median survival ranging from 3 to 12 months. During the last decade there has been significant progress in unravelling the pathophysiology of MPE, as well as its diagnostics, imaging, and management. Nowadays, formerly bed-ridden patients are genotyped, phenotyped, and treated on an ambulatory basis. This article attempts to provide a comprehensive overview of current advances in MPE from bench to bedside. In addition, it highlights unanswered questions in current clinical practice and suggests future directions for basic and clinical research in the field.

@ERSpublications

This review provides up to date knowledge for malignant pleural effusion covering aspects from bench to bedside http://ow.ly/10w7vN

\section{Introduction}

Malignant pleural effusion (MPE) is defined as the accumulation of a significant amount of exudate in the pleural space, accompanied by the presence of malignant cells or tumour tissue. MPE presents a severe medical condition which can result in breathlessness, pain, cachexia and reduced physical activity.

Editorial comment in Eur Respir Rev 2016; 25: 108-109.

Other articles in this series: No. 2: Bhatnagar R, Corcoran JP, Maldonado F, et al. Advanced medical interventions in pleural disease. Eur Respir Rev 2016; 25: 000-000.

Received: Feb 262016 | Accepted after revision: March 252016

Support statement: I. Psallidas is the recipient of a REPSIRE2 European Respiratory Society Fellowship (RESPIRE2 2015-7160). G.T. Stathopoulos is a recipient of European Research Council 2010 Starting Independent Investigator and 2015 Proof of Concept Grants (\#260524 and 679345, respectively). Funding information for this manuscript has been deposited with FundRef.

Conflict of interest: Disclosures can be found alongside the online version of this article at err.ersjournals.com

Provenance: Submitted article, peer reviewed.

Copyright (CERS 2016. ERR articles are open access and distributed under the terms of the Creative Commons Attribution Non-Commercial Licence 4.0. 
Epidemiological information is limited, but an estimated 50000 new diagnoses of MPE are made in the UK each year [1]. The incidence and associated healthcare costs of MPE are expected to rise due to an increase in the global cancer rate and advances in systemic therapy, the latter of which allow many patients to live longer [2].

The majority of MPE is caused by metastatic disease: most commonly lung cancer in men and breast cancer in women. These two cancers combined account for 50-65\% of all MPE [3]. Mesothelioma is the most common type of primary pleural tumour and is associated with MPE in more than $90 \%$ of cases [4]. Despite the progress in cancer treatment, the management of MPE remains palliative, with median survival ranging from 3 to 12 months [5]. Patients' prognosis is highly variable and depends on several factors (primary cancer, stage, performance status and pleural fluid proteins). To this end, the LENT (pleural fluid lactate dehydrogenase, Eastern Cooperative Oncology Group performance score, neutrophil-to-lymphocyte ratio and tumour type) prognostic score was recently proposed as a tool for the accurate prediction of survival [6]. Although LENT has the potential to aid in the assessment of patients with MPE, it requires prospective validation.

During the last decade, there has been significant progress in unravelling the pathophysiology of MPE, as well as its diagnostics, imaging and management. Expanding interest in this area has resulted in an increasing number of cutting-edge laboratory studies that have developed experimental models of MPE which shed light on the pathogenesis of the phenomenon and tested novel biological agents as potential treatment options [7]. Advances in high-throughput techniques (proteomics, genomics) have triggered developments in the discovery of biomarkers for the disease. The use of thoracic ultrasound (TUS) in everyday practice has enhanced the diagnosis of MPE and assisted in the refinement of pleural procedures [8]. Additionally, clinical research in MPE has progressed and high-quality, suitably powered, randomised controlled trials have begun to provide a more robust evidence base for some of the treatment approaches in the field $[9,10]$. This article attempts to provide a comprehensive overview of current advances in MPE from bench to bedside. In addition, it highlights unanswered questions haunting current clinical practice and defines some future directions for basic and clinical research in the field.

\section{Pathophysiology of MPE}

Pleural localisation and propagation of malignant disease

Autopsy studies indicate that tumour cells metastasise to the pleura mainly through the blood-stream and initially invade the visceral pleura $[11,12]$. Most lung carcinomas translocate to the ipsilateral visceral pleura via the pulmonary vessels [11]. Thereafter, secondary dissemination to the parietal pleural occurs by tumour seeding along adhesions or by exfoliated tumour cells floating in the effusion. The pleura may also be invaded through lymphangitic spread or even through direct extension of tumours infiltrating adjacent structures (i.e. lung, chest wall, mediastinum or diaphragm).

For the establishment of viable tumour foci on pleural surface, cancer cells contained in the fluid need to adhere to the mesothelium, escape local anti-tumour immune responses, invade the pleural tissue and obtain access to nutrients and growth stimuli. Tumour-mesothelial cell interactions and cancerous invasion of the pleura implicate adhesion molecules and proteolyic enzymes, although these processes are poorly investigated [13]. Mesothelial cells have been found to limit tumour cell proliferation, whereas the impact of cancerous cells on mesothelial cell growth is controversial. Co-culture studies demonstrated that they either stimulate proliferation or induce apoptosis of mesothelial cells, while in vivo observations suggest that mesothelial cells in MPEs had lower proliferation compared with those in benign effusions [14-16]. Cytokines that possess immune-inhibitory properties and are abundant in the malignancy-affected pleural cavity, as well as local accumulation of immunosuppressive and protumour $\mathrm{CD} 4^{+}$lymphocytes, contribute to immune evasion and facilitate tumour growth [17-21]. Relative to this, MPE is characterised by defective recruitment, activation and the cytotoxic potential of $\mathrm{CD}^{+}$cells $[22,23]$. Macrophages from malignant effusions not only display reduced cytotoxic activity against autologous tumour cells but they also inhibit tumour cell apoptosis [24, 25].

After their detachment from a pleural tumour, cancer cells are deprived of the nutrients provided by the tumour vasculature and of the supportive cell-cell and cell-matrix interactions, thus becoming amenable to apoptosis [26]. However, floating tumour cells not only survive, but are still capable of forming secondary foci in other sites of the pleural cavity denoting that they either dramatically change their metabolic needs or use alternative sources of energy and growth factors. It is therefore possible, though still speculative, that pleural fluid that contains both nutrients and mitogenic/survival stimulators might support cancer cells from the time they exfoliate from a pleural tumour mass until they form secondary implantations that invade the subpleural tissues and obtain access to the host vasculature.

\section{Formation of MPE: general aspects}

Pleural fluid accumulates when its production overwhelms removal. The reason why some tumours cause effusions while others do not is unclear. Post mortem studies demonstrate that mediastinal lymph node 
invasion, but not the extent of pleural involvement, predicts the presence of an effusion [11]. In addition, it has long been believed that pleural fluid clearance occurs through the lymphatics that originate from the stomata of the parietal pleura and drains through the mediastinal nodes. Based on the above, it was initially assumed that impaired pleural fluid drainage, secondary to tumour invasion of the drainage system, is the primary mechanism of MPE formation. However, this notion was challenged by the following: 1) the rate of MPE accumulation is commonly higher than that expected if it is merely due to clearance of the fluid occurring secondary to lymphatic blockage; 2) in the majority of MPE, the protein content is higher than that in normal pleural fluid, suggesting the presence of plasma leakage; and 3) MPE occurs even in patients without parietal pleura involvement. It is therefore currently believed that a combination of increased fluid production due to fluid extravasation from hyper-permeable parietal or visceral pleural and/or tumour vessels and impaired lymphatic outflow underlie the development of MPE [7].

\section{Cell and molecular biology of MPE}

Since pleural fluid overproduction has been recognised as a key event in MPE formation, considerable effort has been invested in unravelling the molecular mechanisms of pleural tumour-driven fluid extravasation. Evidence from in vivo studies indicates that MPE formation is dictated by a complex tumour-host interplay which through paracrine and autocrine effects stimulates pleural inflammation, tumour angiogenesis and vascular hyperpermeability [7]. To trigger these events, tumour cells execute pro-inflammatory and pro-angiogenic transcriptional programmes which are controlled, at least in part, by transcription factors nuclear factor (NF) $-\kappa B$ [27] and signal transducer and activator of transcription (STAT) 3 [28, 29]. Autocrine tumour necrosis factor (TNF), interleukin (IL)-6 and osteopontin (OPN) participate in positive feedback loops regulating tumour NF- $\mathrm{KB} / \mathrm{STAT} 3$ activation to promote MPE [29-31]. In addition, tumour-derived mediators, including vascular endothelial growth factor (VEGF), C-C-motif chemokine ligand (CCL) 2 and TNF, directly stimulate inflammatory cell influx and/or vascular changes [31-34]. Finally, host mediators perpetuate this malicious interplay by amplifying inflammatory and angiogenic signalling loops. To this end, host IL-5 promotes MPE by facilitating the pleural influx of eosinophils and tumour-promoting myeloid suppressor cells [35]. In addition, tumour-expressed OPN protects tumour cells from pro-apoptotic stimuli and host-secreted OPN promotes pleural inflammation and angiogenesis, while the cytokine from both origins provokes vascular leakage [30]. Recently, it has been proved that mast cells are required for MPE formation and by releasing tryptase $\mathrm{AB} 1$ and IL-1 $\beta$ they induce pleural vasculature leakiness and trigger NF- $\mathrm{\kappa B}$ activation thereby fostering fluid accumation and tumour growth [34]. This novel concept on MPE pathogenesis was exploited in preclinical studies that demonstrated that a variety of pharmacological agents including VEGF, sulindac derivative, TNF and angiopoietin inhibitors, zolendronic acid, a dual VEGF receptor/sTie2 antagonist, bortezomib and endostatin curtail experimental MPE, and may now warrant clinical testing [33, 36-40].

\section{Diagnosis \\ Imaging}

The role of imaging techniques is firmly established in the diagnostic workup of patients with suspected MPE. Nowadays, TUS is routinely used by respiratory physicians mainly for the guidance of pleural interventions to minimise complications [41]. The utility of the technique is strongly recommended by national and international guidelines [42]. Evidence also shows that TUS could provide important information on the diagnostic pathway of pleural effusion. Pleural or diaphragmatic thickening and nodularity on TUS are highly specific for malignancy and may therefore help to expedite timely investigation in those with these high-risk features $[8,43]$.

Contrast-enhanced thoracic computed tomography is the current gold-standard imaging modality for the pleura. It can also provide useful information on the pleural cavity as a whole as well as on the primary tumour site and stage. LeUNG et al. [44] suggested that the presence of nodular thickening, mediastinal, parietal and circumferential pleural thickening to have high specificity (88-94\%) but low sensitivity (36-51\%) in identifying malignancy. However, this modality is not perfect and data from a recent retrospective series suggest that approximately one in three patients with pleural malignancy may not demonstrate clear features of cancer on computed tomography [45]. Therefore, invasive investigation and careful follow-up are often warranted even if specific malignant characteristics are not identified in initial imaging studies. The role of positron emission tomography using 18-fluorodeoxyglucose for diagnosing MPE is still not well established. According to a recent meta-analysis of 14 studies including a total of 639 patients, the moderate accuracy of the technique precludes its everyday use to differentiate malignant from benign pleural effusions (table 1) [46].

\section{Cytology-pathology}

The methods used to definitively diagnose MPE have also evolved in recent years. Cytology is a well-established initial test with a mean sensitivity of $60 \%$, but this depends on the underlying primary tumour, sample preparation and experience of the cytologist [47]. The diagnostic yield of pleural fluid 
TABLE 1 Diagnostic accuracy of FDG-PET in the identification of malignant pleural effusions

$\begin{array}{ccccc}\begin{array}{c}\text { Qualitative or } \\ \text { visual PET } \\ \text { readings }\end{array} & \begin{array}{c}\text { Semi-quantitative } \\ \text { (SUV) PET readings }\end{array} & \begin{array}{c}\text { Qualitative or } \\ \text { visual readings } \\ \text { using PET systems }\end{array} & \begin{array}{c}\text { Qualitative readings } \\ \text { using integrated }\end{array} & \begin{array}{c}\text { Semi-quantitative (SUV) } \\ \text { readings using integrated }\end{array} \\ & & \text { PET-CT systems } & \text { PET-CT systems }\end{array}$

\begin{tabular}{lccccc}
\hline Studies n & 11 & 7 & 6 & 5 & 6 \\
Sensitivity \% & 91 & 82 & 96 & 89 & 81 \\
Specificity \% & 67 & 74 & 75 & 61 & 74 \\
Positive LR & 2.83 & 3.24 & 4.09 & 2.32 & 3.22 \\
Negative LR & 0.14 & 0.25 & 0.06 & 0.19 & 0.26 \\
\end{tabular}

FDG: fluorodeoxyglucose; PET: positron emission tomography; SUV: standardised uptake value; CT: computed tomography; LR: likelihood ratio. Data from [46].

cytology for mesothelioma is even lower and most international guidelines advocate the use of pleural biopsy as a preferred diagnostic method over fluid cytology, though the latter is sometimes sufficient in some experienced laboratories [48-51]. In conjunction with the above limitations of cytology, the expanding use of cancer therapies targeted to tissue-specific gene expression or receptor status which necessitates the acquisition of ample tumour tissue for immunohistochemistry and/or genotyping, has led to an increasing requirement for adequately-sized pleural biopsies [52].

Pleural biopsies can be obtained by different techniques: blind, radiologically-guided or through direct visualisation of the pleura. Based on the results of a randomised trial, blind pleural biopsies are no longer advocated given their lower diagnostic yield [53]. Over the recent years, medical thoracoscopy has been increasingly used by respiratory physicians for the investigation of suspected malignant pleural effusions with negative fluid cytology. Medical thoracoscopy has the advantage of a high diagnostic yield and is well established as a key diagnostic test in the investigation of an exudative pleural effusion of unknown cause. The technique can be performed under local anaesthesia with either rigid or semi-rigid scopes and yields a sensitivity of more than $90 \%$ for MPE [54]. Medical thoracoscopy has a favourable safety profile and is considered an overall safe procedure. The BTS reported 16 cases of death in 4736 cases across 47 studies, a mortality rate of $0.3 \%$ [1]. Another advance in pleural sampling is the development of physician-based ultrasound-guided pleural biopsies. Evidence suggests that on frail patients with tethered lungs who may be unfit for medical thoracoscopy, ultrasound-guided pleural biopsy is a valid alternative with a diagnostic yield of $94 \%$ [55].

\section{Biomarkers}

During the previous decade, research has mainly focused on pleural fluid proteins as biomarkers in order to obtain information on patients' diagnosis, prognosis or treatment outcomes. More recently, the development of high-throughput techniques has boosted the characterisation of nucleic acids in MPE. A plethora of different biomarkers have been measured and these include proteins expressed by cancer cells (e.g. mesothelin, CEA, CA15-3, CA125, CYFRA 21-1), surface receptors on immune cells (e.g. CD163 ${ }^{+}$on macrophages), extracellular matrix proteins (e.g. OPN, fibulin-3) and RNA/DNA levels and sequence [56-60]. Although the majority of nucleic acid biomarkers have not yet gained acceptance in clinical practice, analysis of epidermal growth factor receptor (EGFR), EML4-ALK and KRAS mutations have a valid role for the targeted treatment of patients with lung cancer. Interestingly, evidence indicates that EGFR mutations are abundant in patients with MPE compared to primary tumour samples [61].

The clinical implication of diagnostic biomarkers is limited due to the inadequacy of the validation of the results by subsequent studies. Mesothelin, first published as a useful biomarker in 2003, has however since been confirmed as a useful biomarker in serum in at multiple independent studies with very similar sensitivity and specificity levels, and its elevation in pleural fluid is also a useful guide to the presence of malignancy, including in initially cytology negative effusions $[42,62,63]$.

There have been many, often contradictory, biomarker studies with varying specificity and sensitivity, mainly related to the differentiation of malignant from benign effusions [64]. The different results may be ascribed to variations in study populations, measurement techniques (i.e. different ELISA kits for the same cytokine), sample preparation (i.e. measurements in pleural fluid serum versus plasma), failure to include enough control samples in the initial studies or methodological inconsistencies that introduce systematic errors [65]. Promising biomarkers are emerging, but further research with larger studies and prospective validation is required before clinical application. Currently, there are at least two clinical studies pursuing that goal through the prospective collection of samples from patients with MPE (The efficacy of sonographic and 
biological pleurodesis indicators of malignant pleural effusion-SIMPLE; www.isrctn.com ISRCTN16441661) and mesothelioma (Diagnostic and Prognostic Biomarkers in the Rational Assessment of MesotheliomaDIAPHRAGM; www.isrctn.com ISRCTN10079972) using a standardised approach on the timing and testing of biomarkers.

\section{Management}

Although the first randomised trials in MPE were performed in 1977, the optimum management of the disease still remains under debate and research [66]. While the majority of patients with MPE will experience symptoms affecting their quality of life, some remain relatively asymptomatic and can be observed. For those that do develop symptoms, dyspnoea is the most common complaint, followed by chest discomfort and cough [67]. Prior to considering any definitive therapeutic intervention, all patients with MPE should undergo a therapeutic aspiration to assess symptomatic improvement and rate of fluid re-accumulation. Once it is determined that MPE is present, symptomatic and warrants intervention, several options are available.

During the past two decades there has been a change in direction in MPE research and management. Historically, studies were focused on halting pleural fluid accumulation and often employed aggressive surgical methods (pleurectomy); and most clinical trials aimed at identifying the best agent that would achieve obliteration of the pleural space (pleurodesis). The most common end-point of these early studies was radiological improvement at 1-3 months post-pleurodesis, without consideration of the patients' symptoms [68]. Currently, the treatment approach for patients with MPE predominantly aims at alleviating patients' symptoms and improving patient-reported outcome measures, with the latter having gained recognition as a key goal of management [9]. In addition, more aggressive procedures done by thoracic surgeons have been gradually replaced with minimally invasive strategies performed by respiratory physicians. As a result, the ambulatory management of patients with MPE is now more feasible than ever, with indwelling pleural catheters (IPC) and many innovative technologies currently being tested in trials.

\section{Therapeutic aspiration}

In general, selection of the most appropriate treatment approach should be individualised, while also considering patient preference. Patients with poor performance status, minimal life expectancy, and an underlying chemotherapy-sensitive malignancy (e.g., lymphoma or small cell lung cancer) can be managed with therapeutic aspiration [4]. Thoracentesis is generally safe, especially if it is performed under TUS guidance. Re-expansion pulmonary oedema can occur infrequently after the removal of more than $1.5 \mathrm{~L}$ of fluid $(<0.5 \%$ risk in large series) [69]. Although fluid drainage does not improve survival, it can substantially improve symptoms and avoid hospitalisation. The drawback of this approach is that over time the effusion is likely to recur, leading to repeated uncomfortable procedures and relative frequent hospital visits.

\section{Pleurodesis}

Pleurodesis is defined as parietal-to-visceral pleural fusion with concomitant obliteration of the pleural space. It can be accomplished via a variety of chemical or mechanical means, after complete drainage of the effusion that allows parietal-to-visceral pleural apposition. Instillation of the sclerosing agent is thereafter followed by a profound inflammatory response between the layers, which, in turn, result in fibrin accumulation and pleural fibrosis. A variety of different chemicals (talc, bleomycin, tetracycline, iodopovide and others) and bacterial products (from Corynobactum parvum, Streptococcus pyogenes, Staphylococcus aureus and others) have been used in clinical studies to achieve pleurodesis [70-73]. The profound inflammatory response they cause may result in adverse events such as pain and fever but it is believed that the level of inflammation correlates with the likelihood of successful pleurodesis [74].

The type of sclerosant, the method of administration and the method of selection of patients that will be treated with pleurodesis are still unclear. Talc is the most commonly used pleurodesis agent and a meta-analysis supports its use as the sclerosant of choice [4, 75]. Even within the context of large randomised trials, pleurodesis success rates remain under $80 \%$ and are almost certainly lower in everyday practice $[9,10,76,77]$. It has been proven that graded preparations (as opposed to small particle talc) should be used to minimise systemic talc particle dissemination and the risk of acute respiratory distress syndrome $[78,79]$. Talc can be administrated either as a slurry through the chest drain or as poudrage during medical thoracoscopy. Even though it was historically believed that talc poudrage was more effective than slurry, this has changed in light of three randomised studies [76, 80-82]. Results from the largest randomised trial in MPE revealed that the success rates of talc slurry and talc poudrage are not significantly different. A subgroup analysis of patients with lung and breast cancer suggested a better success rate for talc poudrage [76]. On the contrary, a recent metanalysis demonstrated that talc poudrage was superior to talc slurry in pleurodesis success [83]. It is expected that the results of TAPPS trial, which is a suitably powered 
multicentre, open-label, randomised controlled trial designed to compare the pleurodesis success rate of talc poudrage with talc slurry, will provide evidence on the most efficient way of pleurodesis [84].

Studies report a median length of hospital stay of 4-7 days for inpatient talc pleurodesis, which for patients with poorer survival represents a significant proportion of their remaining lives. Even today, no consensus exists about the optimal size of the chest drain and the time of removal of the tube post-pleurodesis. National guidelines advocate the use of small bore $(<14 \mathrm{~F})$ chest drains, but this is now challenged by the results of the largest study to specifically address tube size for MPE in terms of pleurodesis success [10]. Specifically, the TIME1 study showed that smaller chest tubes might be inferior to larger ones in terms of pleurodesis success [10]. The results need validation by future randomised trials powered to address the optimum size of chest drain for pleurodesis.

\section{Indwelling pleural catheters}

IPCs have gained popularity during the last decade as they offer ambulatory management, thereby minimising hospital stay and healthcare costs $[2,74]$. An IPC is a silicone tube placed in the pleural cavity and tunnelled subcutaneously. The proximal end of the exposed tube has a one-way valve which connects to drainage bottles. Drainage is guided by symptoms and is patient-driven, offering a sense of control to most patients. The optimal schedule of MPE drainage through an IPC is still not clear. Two actively recruiting randomised studies (www.clinicaltrials.gov NCT00978939 and NCT00761618) are under way to address this question (aggressive daily versus three times weekly drainage).

Guidelines have advocated the use of IPCs in those patients with MPE that have failed pleurodesis or in those with trapped lung (unsuitable for pleurodesis) [4]. A meta-analysis of 1348 patients with MPE treated with IPCs revealed that $95.6 \%$ had symptomatic improvement and $45.6 \%$ achieved spontaneous pleurodesis after a median of 52 days [85]. In addition, the TIME2 randomised controlled trial showed that IPCs achieved control of breathlessness and quality of life comparable to talc pleurodesis, but significantly shortened the length of hospital stay [9]. Recent data also provided reassurance on the safety of IPC use, with a risk of death from pleural infection below $0.3 \%$ [86]. IPC-related pleural infection occurred in $<5 \%$ of patients enrolled in another multicentre study and was well-controlled with antibiotics. Moreover, patients with an IPC who develop an associated pleural infection appear to have high rates of post-infectious pleurodesis and also prolonged survival times, as suggested by a recent observational study [87]. There is ongoing research on possible combinations of IPC with sclerosant agents in order to enhance pleurodesis success [88]. As IPC offer long-term access to the pleural cavity, they represent ideal potential portals for local drug delivery. In addition to IPC-delivered bacterial moieties that have been evaluated as potential immunomodulators against MPE, IPC will hopefully facilitate further research in this field [82].

\section{Ambulatory management}

Pleural-specific services and technologies are expanding, and this has led to a greater drive towards ambulatory management [89]. The traditional approach of admitting patients with MPE for talc pleurodesis is now outdated. Recent advances in IPC and medical thoracoscopy have created the potential for an attractive "one-stop" approach to diagnosis and management. Combinations of medical thoracoscopy with IPC at the end of the procedure to assure drainage of fluid or sclerosant delivery through IPC could streamline patients' pathways for the limitation of hospitalisation. Clinical trials are currently under way to establish the use and benefit of combined treatment methods.

\section{Future directions}

In vitro assays and animal models of MPE have aided clinicians significantly in better understanding the biology of the condition, and, most likely, have subconsciously contributed to current less invasive ambulatory treatment approaches [7]. These experimental tools should be expanded and improved to accurately reflect the diverse origins and phenotypes of human MPE. It is expected that in future experimental models will be an asset on understanding the cellular origin and components of MPE and will probably result in advancing current treatment options.

Future endeavours in the field of clinical MPE research and management should strive toward individualised and targeted approaches (collectively coined precision medicine), since the disease likely represents a syndrome rather than a robust entity and heterogeneity exists even within different pleural tumour cells from the same patient [90]. In this regard, experimental evidence and clinical observation dictate that MPE development most probably rests on cancer hallmarks and molecular culprits that are distinct from those of tumour metastasis to other organs [7]. Hence, patients with MPE from different primary neoplasms should likely be stratified separately in clinical trials to ensure definitive study outcomes. Even more so, patients with MPE should most certainly be studied alone in clinical trials, and should not be grouped together with patients with other metastatic forms of cancer. To this end, a completely separate stage assigned to MPE in 
the forthcoming revisions of tumour/node/metastases staging systems for lung, breast and other tumours would tremendously augment research in this area.

Whole genome, transcriptome and proteome studies of patients with pleural neoplasms are becoming more feasible every day. Exploitation of these techniques with well-designed experimental setups will likely lead to the identification of the molecular signatures that cause MPE and may also establish malignant effusions as vectors for the liquid molecular biopsy of lung and pleural tumours. In this direction, analyses at single-cell resolution will contribute the most towards identifying the signature of tumour clones that are causative of effusion formation and towards understanding the clonal evolution of pleural cancer dissemination. Novel techniques, such as massive parallel whole transcriptome sequencing of single cells captured within lipid droplets (drop-SEQ), are likely going to revolutionise our capacity to look at the global cancer genome and transcriptome of an unprecedented multitude of single cells from patients with MPE [91].

The implementation of newer management techniques for patients with MPE, such as IPC, should be optimised and will likely constitute a powerful, first-line clinical and research tool in this patient population. It is conceivable that a multitude of longitudinal measurements will be facilitated by wide-spread use of IPC [92]. It is also conceivable that IPC are expected to provide a powerful valuable tool for local therapies against MPE [74].

However, the insights gained into the origins and mechanisms of malignant pleural disease from cell, animal and human studies described will be wasted if they are not directly converted into improvements in patient management and outcome. To this end, better understanding of the molecular culprits of effusion development, as well as the underlying neoplasms, should foster clinical studies of targeted therapies against MPE in the near future [93]. As these molecular alterations are expected to be different in subgroups or even in each individual patient with MPE, targeted therapies will need to be individualised. Parallel co-clinical trials of patient-derived xenografts in humanised mice may direct clinical decisions and help optimise treatment, predict early response, and identify emergent resistance. At the same time that biology is introduced to the clinical field of MPE and palliative treatment is refined, the survival of ambulatory patients with malignant pleural disease is anticipated to change dramatically. Hence, in addition to clinical and molecular assessment, novel prognosticators, such as the LENT score mentioned above, will need to be adjusted to identify sub-phenotypes of MPE with different physical histories. Clinical end-points will also need to be refined during this process. In addition to symptom relief and fluid production, both overall and quality of life-adjusted survival may very well be a meaningful end-point in the near future [94].

\section{Conclusions}

In summary, MPE management and research have made tremendous progress over the last few years. Formerly bed-ridden patients are nowadays genotyped, phenotyped, and treated on an ambulatory basis, and TUS together with IPC have significantly contributed to this change. Medical thoracoscopy is well established as a key diagnostic test in the investigation of MPE. Combined with these powerful clinical tools, adaptations in staging, study end-points and design, as well as application of novel high-throughput research tools to patients with MPE will likely result in etiologic therapy of the condition in the near future.

\section{References}

1 Rahman NM, Ali NJ, Brown G, et al. Local anaesthetic thoracoscopy: British Thoracic Society Pleural Disease Guideline 2010. Thorax 2010; 65: Suppl 2, ii54-ii60.

2 Clive AO, Bhatnagar R, Psallidas I, et al. Individualised management of malignant pleural effusion. Lancet Respir Med 2015; 3: 505-506.

3 Mongardon N, Pinton-Gonnet C, Szekely B, et al. Assessment of chronic pain after thoracotomy: a 1-year prevalence study. Clin J Pain 2011; 27: 677-681.

4 Roberts ME, Neville E, Berrisford RG, et al. Management of a malignant pleural effusion: British Thoracic Society Pleural Disease Guideline 2010. Thorax 2010; 65: Suppl 2, ii32-ii40.

5 Antony VB, Loddenkemper R, Astoul P, et al. Management of malignant pleural effusions. Eur Respir J 2001; 18: 402-419.

6 Clive AO, Kahan BC, Hooper CE, et al. Predicting survival in malignant pleural effusion: development and validation of the LENT prognostic score. Thorax 2014; 69: 1098-1104.

7 Stathopoulos GT, Kalomenidis I. Malignant pleural effusion: tumor-host interactions unleashed. Am J Respir Crit Care Med 2012; 186: 487-492.

8 Qureshi NR, Rahman NM, Gleeson FV. Thoracic ultrasound in the diagnosis of malignant pleural effusion. Thorax 2009; 64: 139-143.

9 Davies HE, Mishra EK, Kahan BC, et al. Effect of an indwelling pleural catheter vs chest tube and talc pleurodesis for relieving dyspnea in patients with malignant pleural effusion: the TIME2 randomized controlled trial. Jama 2012; 307: 2383-2389.

10 Rahman NM, Pepperell J, Rehal S, et al. Effect of opioids versus NSAIDs and larger versus smaller chest tube size on pain control and pleurodesis efficacy among patients with malignant pleural effusion: the TIME1 randomized clinical trial. Jama 2015; 314: 2641-2653.

11 Meyer PC. Metastatic carcinoma of the pleura. Thorax 1966; 21: 437-443. 
12 Rodriguez-Panadero F, Borderas Naranjo F, Lopez Mejias J. Pleural metastatic tumours and effusions. Frequency and pathogenic mechanisms in a post-mortem series. Eur Respir J 1989; 2: 366-369.

13 Antony VB. Immunological mechanisms in pleural disease. Eur Respir J 2003; 21: 539-544.

14 Heath RM, Jayne DG, O'Leary R, et al. Tumour-induced apoptosis in human mesothelial cells: a mechanism of peritoneal invasion by Fas Ligand/Fas interaction. Br J Cancer 2004; 90: 1437-1442.

15 Rehnberg J, Zendehrokh N, Dejmek A. Lower proliferation rate in metastatic effusion mesothelial cells than in benign effusions. Anal Quant Cytol Histol 2007; 29: 217-220.

16 van der Wal BC, Hofland LJ, Marquet RL, et al. Paracrine interactions between mesothelial and colon-carcinoma cells in a rat model. Int J Cancer 1997; 73: 885-890.

17 Chen YM, Yang WK, Whang-Peng J, et al. Elevation of interleukin-10 levels in malignant pleural effusion. Chest 1996; 110: 433-436.

18 Li R, Ruttinger D, Li R, et al. Analysis of the immunological microenvironment at the tumor site in patients with non-small cell lung cancer. Langenbecks Arch Surg 2003; 388: 406-412.

19 Oshikawa K, Yanagisawa K, Tominaga S, et al. Expression and function of the ST2 gene in a murine model of allergic airway inflammation.Clin Exp Allergy 2002; 32: 1520-1526.

20 Ye ZJ, Zhou Q, Yin W, et al. Interleukin 22-producing CD4+ T cells in malignant pleural effusion. Cancer Lett 2012; 326: 23-32.

21 Ye ZJ, Zhou Q, Yuan ML, et al. Differentiation and recruitment of IL-22-producing helper T cells stimulated by pleural mesothelial cells in tuberculous pleurisy. Am J Respir Crit Care Med 2012; 185: 660-669.

22 Prado-Garcia H, Aguilar-Cazares D, Flores-Vergara H, et al. Effector, memory and naive CD8+ $\mathrm{T}$ cells in peripheral blood and pleural effusion from lung adenocarcinoma patients. Lung Cancer 2005; 47: 361-371.

23 Scherpereel A, Grigoriu BD, Noppen M, et al. Defect in recruiting effector memory CD8+ T-cells in malignant pleural effusions compared to normal pleural fluid. BMC Cancer 2013; 13: 324.

24 Pace E, Di Sano C, Ferraro M, et al. Altered CD94/NKG2A and perforin expression reduce the cytotoxic activity in malignant pleural effusions. Eur J Cancer 2011; 47: 296-304.

25 Yanagawa E, Uchida A, Moore $\mathrm{M}$, et al. Autologous tumor killing and natural cytotoxic activity of tumor-associated macrophages in cancer patients. Cancer Immunol Immunother 1985; 19: 163-167.

26 Light RW, Hamm H. Malignant pleural effusion: would the real cause please stand up? Eur Respir J 1997; 10: 1701-1702.

27 Jongsma J, van Montfort E, Vooijs M, et al. A conditional mouse model for malignant mesothelioma. Cancer Cell 2008; 13: 261-271.

28 Stathopoulos GT, Zhu Z, Everhart MB, et al. Nuclear factor-kappaB affects tumor progression in a mouse model of malignant pleural effusion. Am J Respir Cell Mol Biol 2006; 34: 142-150.

29 Yeh HH, Lai WW, Chen HH, et al. Autocrine IL-6-induced Stat3 activation contributes to the pathogenesis of lung adenocarcinoma and malignant pleural effusion. Oncogene 2006; 25: 4300-4309.

30 Psallidas I, Stathopoulos GT, Maniatis NA, et al. Secreted phosphoprotein-1 directly provokes vascular leakage to foster malignant pleural effusion. Oncogene 2013; 32: 528-535.

31 Stathopoulos GT, Kollintza A, Moschos C, et al. Tumor necrosis factor-alpha promotes malignant pleural effusion. Cancer Res 2007; 67: 9825-9834.

32 Stathopoulos GT, Psallidas I, Moustaki A, et al. A central role for tumor-derived monocyte chemoattractant protein-1 in malignant pleural effusion. J Natl Cancer Inst 2008; 100: 1464-1476.

33 Yano S, Shinohara H, Herbst RS, et al. Production of experimental malignant pleural effusions is dependent on invasion of the pleura and expression of vascular endothelial growth factor/vascular permeability factor by human lung cancer cells. Am J Pathol 2000; 157: 1893-1903.

34 Giannou AD, Marazioti A, Spella M, et al. Mast cells mediate malignant pleural effusion formation. J Clin Invest 2015; 125: 2317-2334.

35 Stathopoulos GT, Sherrill TP, Karabela SP, et al. Host-derived interleukin-5 promotes adenocarcinoma-induced malignant pleural effusion. Am J Respir Crit Care Med 2010; 182: 1273-1281.

$36 \mathrm{Ma} \mathrm{X}$, Yao Y, Yuan D, et al. Recombinant human endostatin endostar suppresses angiogenesis and lymphangiogenesis of malignant pleural effusion in mice. PloS One 2012; 7: e53449.

37 Moschos C, Psallidas I, Cottin T, et al. A sulindac analogue is effective against malignant pleural effusion in mice. Lung Cancer 2011; 73: 171-175.

38 Moschos C, Psallidas I, Kollintza A, et al. The angiopoietin/Tie2 axis mediates malignant pleural effusion formation. Neoplasia 2009; 11: 298-304.

39 Psallidas I, Karabela SP, Moschos C, et al. Specific effects of bortezomib against experimental malignant pleural effusion: a preclinical study. Mol Cancer 2010; 9: 56

40 Stathopoulos GT, Moschos C, Loutrari $\mathrm{H}$, et al. Zoledronic acid is effective against experimental malignant pleural effusion. Am J Respir Crit Care Med 2008; 178: 50-59.

41 Gordon CE, Feller-Kopman D, Balk EM, et al. Pneumothorax following thoracentesis: a systematic review and meta-analysis. Arch Intern Med 2010; 170: 332-339.

42 Havelock T, Teoh R, Laws D, et al. Pleural procedures and thoracic ultrasound: British Thoracic Society Pleural Disease Guideline 2010. Thorax 2010; 65: Suppl 2, ii61-ii76.

43 Bugalho A, Ferreira D, Dias SS, et al. The diagnostic value of transthoracic ultrasonographic features in predicting malignancy in undiagnosed pleural effusions: a prospective observational study. Respiration 2014; 87: $270-278$.

44 Leung AN, Muller NL, Miller RR. CT in differential diagnosis of diffuse pleural disease. AJR Am J Roentgenol 1990; 154: 487-492.

45 Hallifax RJ, Haris M, Corcoran JP, et al. Role of CT in assessing pleural malignancy prior to thoracoscopy. Thorax 2014.

46 Porcel JM, Hernandez P, Martinez-Alonso M, et al. Accuracy of fluorodeoxyglucose-PET imaging for differentiating benign from malignant pleural effusions: a meta-analysis. Chest 2015; 147: 502-512.

47 Hooper C, Lee YC, Maskell N, et al. Investigation of a unilateral pleural effusion in adults: British Thoracic Society Pleural Disease Guideline 2010. Thorax 2010; 65: Suppl 2, ii4-ii17.

48 Renshaw AA, Dean BR, Antman KH, et al. The role of cytologic evaluation of pleural fluid in the diagnosis of malignant mesothelioma. Chest 1997; 111: 106-109. 
49 van Zandwijk N, Clarke C, Henderson D, et al. Guidelines for the diagnosis and treatment of malignant pleural mesothelioma. J Thorac Dis 2013; 5: E254-E307.

50 Scherpereel A, Astoul P, Baas P, et al. Guidelines of the European Respiratory Society and the European Society of Thoracic Surgeons for the management of malignant pleural mesothelioma. Eur Respir J 2010; 35: 479-495.

51 Segal A, Sterrett GF, Frost FA, et al. A diagnosis of malignant pleural mesothelioma can be made by effusion cytology: results of a 20 year audit. Pathology 2013; 45: 44-48.

52 Henderson DW, Reid G, Kao SC, et al. Challenges and controversies in the diagnosis of mesothelioma: Part 1. Cytology-only diagnosis, biopsies, immunohistochemistry, discrimination between mesothelioma and reactive mesothelial hyperplasia, and biomarkers. J Clin Pathol 2013; 66: 847-853.

53 Maskell NA, Gleeson FV, Davies RJO. Standard pleural biopsy versus CT-guided cutting-needle biopsy for diagnosis of malignant disease in pleural effusions: a randomised controlled trial. Lancet 2003; 361: 1326-1330

54 Menzies R, Charbonneau M. Thoracoscopy for the diagnosis of pleural disease. Ann Intern Med 1991; 114: 271-276.

55 Hallifax RJ, Corcoran JP, Ahmed A, et al. Physician-based ultrasound-guided biopsy for diagnosing pleural disease. Chest 2014; 146: 1001-1006.

56 Creaney J, Dick IM, Robinson BW. Comparison of mesothelin and fibulin-3 in pleural fluid and serum as markers in malignant mesothelioma. Curr Opin Pulm Med 2015; 21: 352-356.

57 Pass HI, Lott D, Lonardo F, et al. Asbestos exposure, pleural mesothelioma, and serum osteopontin levels. $N$ Engl J Med 2005; 353: 1564-1573.

58 Porcel JM, Vives M, Esquerda A, et al. Use of a panel of tumor markers (carcinoembryonic antigen, cancer antigen 125, carbohydrate antigen 15-3, and cytokeratin 19 fragments) in pleural fluid for the differential diagnosis of benign and malignant effusions. Chest 2004; 126: 1757-1763.

59 Creaney J, Yeoman D, Demelker Y, et al. Comparison of osteopontin, megakaryocyte potentiating factor, and mesothelin proteins as markers in the serum of patients with malignant mesothelioma. J Thorac Oncol 2008; 3: 851-857.

60 Pass HI, Levin SM, Harbut MR, et al. Fibulin-3 as a blood and effusion biomarker for pleural mesothelioma. N Engl J Med 2012; 367: 1417-1427.

61 Tsai TH, Su KY, Wu SG, et al. RNA is favourable for analysing EGFR mutations in malignant pleural effusion of lung cancer. Eur Respir J 2012; 39: 677-684.

62 Robinson BW, Creaney J, Lake R, et al. Mesothelin-family proteins and diagnosis of mesothelioma. Lancet 2003; 362: 1612-1616.

63 Creaney J, Segal A, Olsen N, et al. Pleural fluid mesothelin as an adjunct to the diagnosis of pleural malignant mesothelioma. Dis Markers 2014; 2014: 413946.

64 Sriram KB, Relan V, Clarke BE, et al. Diagnostic molecular biomarkers for malignant pleural effusions. Future Oncology 2011; 7: 737-752.

65 Blyth KG. Inconsistent results or inconsistent methods? A plea for standardisation of biomarker sampling in mesothelioma studies. Thorax 2015; 70: 374.

66 Mejer J, Mortensen KM, Hansen HH. Mepacrine hydrochloride in the treatment of malignant pleural effusion. A controlled randomized trial. Scand J Respir Dis 1977; 58: 319-323.

67 Martinez-Moragon E, Aparicio J, Sanchis J, et al. Malignant pleural effusion: prognostic factors for survival and response to chemical pleurodesis in a series of 120 cases. Respiration 1998; 65: 108-113.

68 Davies HE, Lee YC. Management of malignant pleural effusions: questions that need answers. Curr Opin Pulm Med 2013; 19: 374-379.

69 Feller-Kopman D, Berkowitz D, Boiselle P, et al. Large-volume thoracentesis and the risk of reexpansion pulmonary edema. Ann Thorac Surg 2007; 84: 1656-1661.

70 Agarwal R, Paul AS, Aggarwal AN, et al. A randomized controlled trial of the efficacy of cosmetic talc compared with iodopovidone for chemical pleurodesis. Respirology 2011; 16: 1064-1069.

71 Kishi K, Homma S, Sakamoto S, et al. Efficacious pleurodesis with OK-432 and doxorubicin against malignant pleural effusions. Eur Respir J 2004; 24: 263-266.

72 Ren S, Terman DS, Bohach G, et al. Intrapleural staphylococcal superantigen induces resolution of malignant pleural effusions and a survival benefit in non-small cell lung cancer. Chest 2004; 126: 1529-1539.

73 Ukale V, Agrenius V, Hillerdal G, et al. Pleurodesis in recurrent pleural effusions: a randomized comparison of a classical and a currently popular drug. Lung Cancer 2004; 43: 323-328.

74 Thomas R, Francis R, Davies HE, et al. Interventional therapies for malignant pleural effusions: the present and the future. Respirology 2014; 19: 809-822.

75 Shaw P, Agarwal R. Pleurodesis for malignant pleural effusions. Cochrane Database Syst Rev 2004; CD002916.

76 Dresler CM, Olak J, Herndon JEII, et al. Phase III intergroup study of talc poudrage vs talc slurry sclerosis for malignant pleural effusion. Chest 2005; 127: 909-915.

77 Rintoul RC, Ritchie AJ, Edwards JG, et al. Efficacy and cost of video-assisted thoracoscopic partial pleurectomy versus talc pleurodesis in patients with malignant pleural mesothelioma (MesoVATS): an open-label, randomised, controlled trial. Lancet 2014; 384: 1118-1127.

78 Janssen JP, Collier G, Astoul P, et al. Safety of pleurodesis with talc poudrage in malignant pleural effusion: a prospective cohort study. Lancet 2007; 369: 1535-1539.

79 Maskell NA, Lee YC, Gleeson FV, et al. Randomized trials describing lung inflammation after pleurodesis with talc of varying particle size. Am J Respir Crit Care Med 2004; 170: 377-382.

80 Terra RM, Junqueira JJ, Teixeira LR, et al. Is full postpleurodesis lung expansion a determinant of a successful outcome after talc pleurodesis? Chest 2009; 136: 361-368.

81 Yim AP, Chan AT, Lee TW, et al. Thoracoscopic talc insufflation versus talc slurry for symptomatic malignant pleural effusion. Ann Thorac Surg 1996; 62: 1655-1658.

82 Rahman NM, Davies HE, Salzberg M, et al. Use of lipoteichoic acid-T for pleurodesis in malignant pleural effusion: a phase I toxicity and dose-escalation study. Lancet Oncol 2008; 9: 946-952.

83 Xia H, Wang XJ, Zhou Q, et al. Efficacy and safety of talc pleurodesis for malignant pleural effusion: a meta-analysis. PloS One 2014; 9: e87060.

84 Bhatnagar R, Laskawiec-Szkonter M, Piotrowska HE, et al. Evaluating the efficacy of thoracoscopy and talc poudrage versus pleurodesis using talc slurry (TAPPS trial): protocol of an open-label randomised controlled trial. BMJ Open 2014; 4: e007045. 
85 Van Meter ME, McKee KY, Kohlwes RJ. Efficacy and safety of tunneled pleural catheters in adults with malignant pleural effusions: a systematic review. J Gen Intern Med 2011; 26: 70-76.

86 Fysh ET, Tremblay A, Feller-Kopman D, et al. Clinical outcomes of indwelling pleural catheter-related pleural infections: an international multicenter study. Chest 2013; 144: 1597-1602.

87 Bibby AC, Clive AO, Slade GC, et al. Survival in patients with malignant pleural effusions who developed pleural infection: a retrospective case review from six UK centers. Chest 2015; 148: 235-241.

88 Bhatnagar R, Kahan BC, Morley AJ, et al. The efficacy of indwelling pleural catheter placement versus placement plus talc sclerosant in patients with malignant pleural effusions managed exclusively as outpatients (IPC-PLUS): study protocol for a randomised controlled trial. Trials 2015; 16: 48.

89 Bhatnagar R, Maskell N. Developing a 'pleural team' to run a reactive pleural service. Clin Med 2013; 13: 452-456.

90 Basak SK, Veena MS, Oh S, et al. The malignant pleural effusion as a model to investigate intratumoral heterogeneity in lung cancer. PloS One 2009; 4: e5884.

91 Macosko EZ, Basu A, Satija R, et al. Highly parallel genome-wide expression profiling of individual cells using nanoliter droplets. Cell 2015; 161: 1202-1214.

92 Thomas R, Cheah HM, Creaney J, et al. Longitudinal measurement of pleural fluid biochemistry and cytokines in malignant pleural effusions. Chest 2016 [in press; DOI: 10.1016/j.chest.2016.01.001].

93 Tsai TH, Wu SG, Chang YL, et al. Effusion immunocytochemistry as an alternative approach for the selection of first-line targeted therapy in advanced lung adenocarcinoma. J Thorac Oncol 2012; 7: 993-1000.

94 Ost DE, Jimenez CA, Lei X, et al. Quality-adjusted survival following treatment of malignant pleural effusions with indwelling pleural catheters. Chest 2014; 145: 1347-1356. 